\title{
EFECTO DE APLICACIÓN DE DIFERENTES LÁMINAS DE RIEGO EN ESTEVIA (Stevia rebaudiana Bert.) BAJO INVERNADERO
}

\section{THE EFFECT OF APPLYING DIFFERENT WATER LEVELS IN STEVIA}

\author{
Martha C. Daza ${ }^{1 *}$, Jorge A. Jurado² y Victor M. Torres ${ }^{3}$
}

Recibido para publicación: Enero 21 de 2015 - Aceptado para publicación: Mayo 20 de 2015

\begin{abstract}
RESUMEN
La determinación de adecuadas láminas de riego es importante para el manejo del agua en la agricultura y para garantizar la sostenibilidad de los sistemas productivos. El objetivo de este trabajo fue evaluar diferentes láminas de riego calculadas a partir de coeficientes multiplicadores de la evapotranspiración, aplicadas a un cultivo de Estevia (Stevia Rebaudiana Bert.). Se utilizó un diseño completamente aleatorio realizado en condiciones controladas con 5 tratamientos correspondientes a los coeficientes multiplicadores de la evapotranspiración $(0,9,1,0,1,1,1,2$ y 1,3$)$ a partir de los cuales fueron calculadas las láminas de riego que fueron aplicadas a las plantas. Cada tratamiento tuvo cinco repeticiones para un total de 25 unidades experimentales. Se evaluaron el número de hojas por planta, el peso fresco y seco, el porcentaje de materia seca, la longitud de raíz, el diámetro de tallo, el contenido relativo de agua en las hojas y el rendimiento del cultivo. No se encontraron diferencias significativas entre coeficientes para las diferentes variables agronómicas evaluadas. Sin embargo, se observó la tendencia de que el coeficiente de 1,2 mostró los mejores resultados y puede considerarse como valor guía para el manejo del agua en este cultivo en zonas donde no se cuenta con estudios de coeficientes de cultivo.
\end{abstract}

Palabras Clave: Coeficiente de cultivo, recurso hídrico, cultivos aromáticas.

\begin{abstract}
Determination of appropriate irrigation levels is important for water management in agriculture and for ensuring the sustentability of the production systems. The objective of this study was to evaluate different irrigation levels, calculated from multiplier evapotranspiration coefficients, applied to a stevia (Stevia rebaudiana B) crop. A completely randomized design, with 5 treatments and five repetitions by treatments, was conducted under controlled conditions. Each treatment consisted of a water irrigation level estimated using different evapotranspiration multipliers $(0,9 ; 1,0 ; 1,1 ; 1,2$ and 1,3$)$. In each plant, the number of leaves, fresh and dry weight, dry matter, root length, stem diameter, relative water content in leaves and crop yield were evaluated.

\footnotetext{
${ }^{1}$ Ingeniera Agrícola M Sc en Suelos y Aguas. Candidata a Doctor en Ingeniería Sanitaria y Ambiental. Docente asociada Escuela de Ingeniería de Recursos Naturales y del Ambiente - EIDENAR. Universidad del Valle, Cali, Colombia. calle 42 № 85 E 38 Altos del Caney Cali Valle- mail: martha.daza@correounivalle.edu.co

${ }^{2}$ Ingeniero Agrícola. Investigador auxiliar en la Corporación Centro de Investigación en Palma de Aceite CENIPALMA. Cumaral-Meta Colombia, Calle 25 №36 - 55 La Esperanza Cali Valle,mail: jjurado@cenipalma.org

${ }^{3}$ Ingeniero Agrícola. Instructor de la Tecnología en control ambiental; Centro de Diseño Tecnológico Industrial, SENA-Regional Valle, Cali,
} Colombia. Calle 72K \#26J - 97. Mail: vmtorres05@misena.edu.co, Calle $66 \mathrm{~N}^{\circ} 1$ - 43 Cali Valle, mail: victormanueltorreslozano@gmail.com
\end{abstract}


No significant differences were observed among these variables. However, there was a trend that the best results were observed when the irrigation level was calculated using the 1,2 coefficient of evapotranspiration. Consequently, that coefficient should used for water irrigation management in this crop in areas where crop coefficients studies are not available

Key-words: crop coefficients, water resource, aromatic crops

\section{INTRODUCCIÓN}

La agricultura es una de las actividades económicas que mayor demanda de agua presenta a nivel mundial y el riego de tierras agrícolas estima la utilización entre el 33 y el $90 \%$ de los recursos hídricos en el mundo (EEA 2012; ONU 2011), lo cual lo convierte en una actividad ineficiente y que afecta de manera inmediata el recurso (Martínez 2010). En Colombia las plantas aromáticas y en especial la Estevia son cultivos que aun no han alcanzado altos rendimientos en comparación con otras partes del mundo debido a la falta de desarrollo de paquetes tecnológicos. En efecto, son pocos los estudios de que se disponen acerca del comportamiento agronómico de las plantas aromáticas cuando se someten a diferentes láminas de riego y al estrés hídrico (Silva 2002). Cuervo (2012), asegura que en el caso específico de la Estevia, la susceptibilidad a la sequía durante su etapa vegetativa es alta y el manejo del riego está directamente relacionado con la concentración de sus principios activos.

Gran parte de la responsabilidad del inadecuado manejo del agua obedece al cálculo sobreestimado de las láminas de riego por el desconocimiento del coeficiente del cultivo $(\mathrm{Kc})$. Este coeficiente ha sido definido como la relación entre la evapotranspiración del cultivo y la evapotranspiración potencial
(Allen et al. 2006). Diversos valores de Kc para Estevia han sido hallados en diversas partes del mundo, como los reportados por Fronza y Vinícius (2003) cuyos valores estuvieron entre 1,16 y 1,45 y los reportados por González et al. (2002) quienes en Paraguay encontraron valores más bajos (entre 0,25 y 0,82) mostrando que los valores de Kc dependen de las condiciones ambientales donde se desarrolla el cultivo. En zonas con precipitaciones menores a 1400 $\mathrm{mm}$, se han encontrado necesidades hídricas para Estevia de $5 \mathrm{~mm}$ los cuales recomiendan ser aplicados cada tres días si los suelos son arenosos y cada 5 días si son arcillosos (Casaccia y Álvarez 2006). Esta disparidad en los valores de $\mathrm{Kc}_{\mathrm{c}}$ han hecho que en los sistemas productivos agrícolas, el uso de riego suplementario sea obligatorio (Hadid 2004).

Para determinar los coeficientes del cultivo es necesario realizar balances hídricos haciendo uso de los lisímetros. La medición de la evapotranspiración potencial se estima a partir de la ecuación de Penman Monteith la cual considera parámetros climáticos (Allen et al. 2006) mientras que la evapotranspiración del cultivo se realiza teniendo en cuenta las entradas y salidas de agua del lisímetro y la capacidad de almacenamiento hídrico del suelo. A pesar de que los lisímetros son alternativas de relativa facilidad para su construcción, en países en vías de desarrollo contar con la información climática puede ser un gran reto, ya que la 
probabilidad de existencia de estaciones climatológicas cercanas que contenga la información completa es baja; además, contar con una estación meteorológica portátil no siempre es posible.

Ante estas dificultades se han desarrollado metodologías alternativas como el diseño de experimentos en campo haciendo uso de coeficientes multiplicadores (Alvarez et al. 2010). El coeficiente multiplicador es un valor aproximado de Kc estimado en experimentos en campo, en donde se adiciona láminas de riego determinadas a partir del producto de diversos coeficientes y la evaporación diaria y correlacionándo con el desarrollo de características fisiológicas de las plantas. A pesar de que la metodología no permite obtener valores de Kc puntuales, permite la estimación de valores de coeficientes de cultivo aproximados que permiten hacer manejos más racionales del recurso hídrico.

El objetivo de este trabajo fue evaluar láminas de riego obtenidas a partir de la variación de diferentes coeficientes multiplicadores de la evapotranspiración diaria, en el desarrollo de plantas de Estevia (Stevia rebaudiana Bert.) en condiciones controladas

\section{MATERIALES Y MÉTODOS}

El presente trabajo de investigación se realizó en condiciones de invernadero del Laboratorio de Aguas y Suelos Agrícolas - LASA de la Universidad del Valle localizada en el municipio de Santiago de Cali, Valle del Cauca, con una altura de 979 msnm; coordenadas latitud $3^{\circ} 22^{\prime} 22,29^{\prime \prime} \mathrm{N}$, longitud $76^{\circ} 31^{\prime}$ $49,22^{\prime \prime}$ O. Dentro del invernadero se localizó un tanque evaporímetro construido con las especificaciones de la FAO, (Allen et al. 2006) y calibrado con la estación meteorológica del IDEAM ciudadela Melendez, ubicada dentro de los predios de la Universidad.

El suelo utilizado fue muestreado de la finca "La Hacienda" localizada en la vereda "Agua Azul" en la zona rural del municipio de Villa Rica (Cauca, Colombia), a 36 kilómetros de la ciudad de Cali (Valle del Cauca), con una altura de 982 msnm, una temperatura promedio de $27^{\circ} \mathrm{C}$ y precipitación media de $1850 \mathrm{~mm}$ año ${ }^{-1}$, con latitud $3^{\circ} 18^{\prime} 21^{\prime \prime} \mathrm{N}$ y longitud $76^{\circ} 44^{\prime} 72^{\prime \prime}$. Presentó textura franco arcillosa, reacción moderadamente ácida, con bajo nivel de materia orgánica y sin problemas de salinidad (Tabla 1).

Se utilizó un diseño unifactorial completamente al azar donde el factor coeficiente multiplicador (CM) de la evapotranspiración con 5 niveles $(0,9 ; 1,0 ; 1,1 ; 1,2$ y 1,3$)$ equivalentes a los 5 tratamientos, fue reemplazado en la siguiente ecuación:

Volumen de riego $=\mathrm{EV} \times \mathrm{C} \times \mathrm{CM} \times \mathrm{A}$

Donde EV es la evaporación medida en el tanque evaporímetro; $C$ es el coeficiente del tanque equivalente a 0,55 (Allen et al. 2006); CM es el coeficiente multiplicador equivalente a reemplazar el coeficiente del cultivo y A es el área efectiva calculada a partir del diámetro de la bolsa donde se desarrolló la planta. Se tuvieron 5 repeticiones para un total de 25 unidades experimentales. Cada unidad experimental consistió en una bolsa de polietileno calibre 18 de $4 \mathrm{~kg}$ de capacidad dentro de la cual se colocó el suelo tamizado 
Tabla 1. Caracterización de propiedades químicas y contenido de nutrientes del suelo provenientes de Villa Rica (Cauca - Colombia).

\begin{tabular}{cccccc}
\hline Propiedad & Unidad & Valor & Propiedad & Unidad & Valor \\
\hline Textura & - & $\begin{array}{c}\text { Franco } \\
\text { arenosa }\end{array}$ & $\mathrm{K}$ intercambiable & $\mathrm{cmol}^{(+)} \mathrm{kg}^{-1}$ & 0,18 \\
$\mathrm{pH}$ & - & 5,09 & $\mathrm{Na}$ intercambiable & $\mathrm{cmol}^{(+)} \mathrm{kg}^{-1}$ & 0,04 \\
C.E.* & $\mathrm{dS} \mathrm{m}{ }^{-1}$ & 0,11 & P disponible & $\mathrm{mg} \mathrm{kg}^{-1}$ & 37,90 \\
M.O. & $\%$ & 2,28 & $\mathrm{~B}$ & $\mathrm{mg} \mathrm{kg}^{-1}$ & 0,29 \\
Al intercambiable & $\mathrm{Cmol}^{(+)} \mathrm{kg}^{-1}$ & 0,36 & $\mathrm{Fe}$ & $\mathrm{mg} \mathrm{kg}^{-1}$ & 82,70 \\
$\begin{array}{c}\text { Acidez } \\
\text { Intercambiable }\end{array}$ & $\mathrm{Cmol}^{(+)} \mathrm{kg}^{-1}$ & 0,52 & $\mathrm{Cu}$ & $\mathrm{mg} \mathrm{kg}^{-1}$ & 0,82 \\
CICE** & $\mathrm{Cmol}^{(+)} \mathrm{kg}^{-1}$ & 3,97 & $\mathrm{Mn}$ & $\mathrm{mg} \mathrm{kg}^{-1}$ & 5,98 \\
Ca intercambiable & $\mathrm{Cmol}^{(+)} \mathrm{kg}^{-1}$ & 2,62 & $\mathrm{Zn}$ & $\mathrm{mg} \mathrm{kg}^{-1}$ & 0,48 \\
Mg intercambiable & $\mathrm{Cmol}^{(+)} \mathrm{kg}^{-1}$ & 0,61 & $\mathrm{Si}$ & $\mathrm{mg} \mathrm{kg}^{-1}$ & $\mathrm{~N} . \mathrm{D}$.
\end{tabular}

${ }^{*}$ C.E: $=$ conductividad eléctrica; ${ }^{* *}$ M.O.= Materia orgánica; ${ }^{* * *} \mathrm{CICE}=$ Capacidad de intercambio Catiónico Efectiva.

por $2 \mathrm{~mm}, 0,2 \mathrm{~kg}$ de lombricompost y una planta de Estevia previamente germinada en semillero. Las unidades experimentales se localizaron a $0,10 \mathrm{~m}$ del suelo para evitar la prolongación de raíces hacia el suelo del invernadero.

Al inicio del experimento el suelo fue saturado y se dejó drenar por 48 horas hasta alcanzar la capacidad de campo la cual fue corroborada por medio del tensiómetro y la curva de retención de humedad previamente determinada. Se realizaron mediciones diarias de evaporación y se calculó el volumen diario de aplicación de agua el cual fue aplicado con probeta milimétrica. En el momento del trasplante se aplicó 2,24 g de fertilizante 10 - 30 - 10 (NPK) que fueron distribuidos uniformemente en un volumen de agua de $40 \mathrm{~mL}$.
Se realizaron podas de formación a los 8 y 23 días después del trasplante. A los 62 días después del trasplante se cortó la planta y se determinaron variables de respuesta como número de hojas por planta, peso foliar fresco y seco determinado por secado de la muestra vegetal a $70{ }^{\circ} \mathrm{C}$ por 24 horas, porcentaje de materia seca, longitud de raíz, peso seco de la raíz, diámetro de tallo, área foliar medida de forma indirecta con el peso de la hoja, contenido relativo de agua en las hojas (estado hídrico) medido como la relación entre el contenido de agua presente en la hoja y el máximo contenido de agua que puede almacenar (Argentel et al. 2006) y rendimiento de cultivo.

A los datos obtenidos se les comprobó que siguieran una distribución normal por 
medio de la prueba de Shapiro - Wills y homogeneidad de varianzas por la prueba de Lavene. Se realizó un análisis de varianza para determinar diferencias significativas. Se utilizó el programa R versión 2.15.0 de uso libre para los análisis estadísticos.

\section{RESULTADOS Y DISCUSIÓN}

El número de hojas por planta estuvieron entre 80 y 110 sin diferencias significativas entre tratamientos (Tabla 2). El mayor coeficiente multiplicador obtuvo el mayor número de hojas por planta. De acuerdo con Vera et al. (2001) un mayor consumo de agua promueve mayor absorción de nutrientes como el nitrógeno que participa activamente en el desarrollo de la planta y por tanto en la generación de masa vegetal. Similares resultados fueron encontrados por Marín (2010) quien encontró que al aumentar la cantidad de agua aplicada al cilantro, mayor fue el desarrollo de hojas basales.

A mayor coeficiente multiplicador de la evapotranspiración, mayor fue el peso fresco y seco obtenido y por tanto el porcentaje de materia seca, lo que relaciona la importancia del agua en la absorción de nutrientes esenciales para el crecimiento y desarrollo de las plantas (Tabla 2). Lavini et al. (2008) encontraron que la masa seca total de la planta y de las hojas se reducen conforme el potencial mátrico del suelo aumenta, es decir, la energía que necesita la planta para absorber agua es mayor que si el suelo esta a capacidad de campo. Sin embargo, es conocido que existe que cada especie alcanza un valor máximo del coeficiente del cultivo donde se expresa su máximo desarrollo. En efecto, la aplicación de láminas excesivas de agua, puede ser contraproducente puesto que limita la aireación del suelo creando condiciones anaeróbicas nocivas para las plantas como ha sido reportado por Casierra y Vargas (2007). Jarma et al. (2005) reportaron similares valores de peso foliar por planta los cuales estuvieron por debajo de los $5 \mathrm{~g}$ planta $^{-1}$ evaluadas a los 90 días después del trasplante. Es posible que coeficientes mayores puedan mejorar el desarrollo de las plantas de Estevia. Álvarez et al. (2010) encontraron que a mayor suministro de agua, se incrementaba la actividad de las raíces y por tanto la eficiencia en el transporte de nutrientes a través del xilema y el floema aumentando el crecimiento y desarrollo de la planta de romero.

El valor más bajo de coeficiente multiplicador $(0,9)$ obtuvo la mayor longitud de raíz, debido a la necesidad de las plantas de buscar agua en situaciones de escasez. Los coeficientes 1,$0 ; 1,1$ y 1,3 obtuvieron longitudes de raíces por debajo de 0,10 m. Así mismo el peso seco de raíz fue mayor en el coeficiente más bajo indicando la necesidad de concentración de nutrientes en la raíz de tal manera que permita tener mayor capacidad de exploración de suelo. Sin embargo, no se presentaron diferencias entre coeficientes mostrando resultados similares a los hallados por Wilches y Alvarez (2007) quienes a pesar de variar las láminas de riego en plantas de tomate, no encontraron diferencias significativas ni en longitud ni en el peso seco de raíces.

Los diámetros de tallo estuvieron entre 1,45 y 2,15 mm correspondientes a los coeficientes de 1,0 y 1,3 respectivamente. De acuerdo a los resultados, estos coeficientes multiplicadores 
Tabla 2. Efecto de la lámina de riego calculada a partir de la variación de coeficientes multiplicadores de la evapotransportación en características agronómicas de Estevia(stevia Rebaudiana Bert).

\begin{tabular}{|c|c|c|c|c|c|c|c|}
\hline CM & $\begin{array}{c}\text { Hojas por } \\
\text { planta }\end{array}$ & $\begin{array}{l}\text { Peso foliar } \\
\text { fresco (g) }\end{array}$ & $\begin{array}{l}\text { Peso foliar } \\
\text { seco (g) }\end{array}$ & $\begin{array}{l}\text { Longitud de } \\
\text { raiz }(\mathbf{c m})\end{array}$ & $\begin{array}{l}\text { Peso seco } \\
\text { raiz }(\mathbf{c m})\end{array}$ & $\begin{array}{c}\text { Diámetro de } \\
\text { tallo }(\mathbf{m m})\end{array}$ & $\begin{array}{l}\text { Área foliar } \\
\quad\left(\mathbf{c m}^{2}\right)\end{array}$ \\
\hline 0,9 & 81 & $6,42 \pm 0,62$ & $2,47 \pm 0,24$ & $12,13 \pm 4,93$ & $1,13 \pm 0,47$ & $2,03 \pm 0,17$ & $3,44 \pm 0,60$ \\
\hline 1,0 & 96 & $7,69 \pm 2,23$ & $2,54 \pm 0,73$ & $9,28 \pm 3,52$ & $0,60 \pm 0,37$ & $1,68 \pm 0,51$ & $2,92 \pm 1,24$ \\
\hline 1,1 & 78 & $7,35 \pm 3,25$ & $2,69 \pm 1,19$ & $9,30 \pm 4,99$ & $0,60 \pm 0,23$ & $1,45 \pm 0,57$ & $3,62 \pm 0,63$ \\
\hline 1,2 & 78 & $9,09 \pm 4,08$ & $3,21 \pm 1,45$ & $10,05 \pm 3,51$ & $0,55 \pm 0,24$ & $2,05 \pm 0,40$ & $4,20 \pm 0,87$ \\
\hline 1,3 & 106 & $10,90 \pm 2,85$ & $3,04 \pm 2,05$ & $9,38 \pm 1,11$ & $0,70 \pm 0,14$ & $2,15 \pm 0,29$ & $4,02 \pm 1,28$ \\
\hline $\mathrm{F}$ & 0,81 & 1,5 & 0,26 & 0,39 & 2,25 & 2,01 & 1,08 \\
\hline $\mathrm{p}$ & 0,54 & 0,25 & 0,90 & 0,81 & 0,11 & 2,15 & 0,34 \\
\hline
\end{tabular}

${ }^{*} \mathrm{CM}=$ coeficiente multiplicador. Valores promedio de cuatro repeticiones \pm error estándar. *valores promedio de diez repeticiones \pm error estándar. No se encontraron diferencias significativas con un nivel de confianza del 95\% determinado por el test de Tukey.

aun resultaron bajos en la etapa de crecimiento y madurez que no permitió el desarrollo total de las plantas. Sin embargo, estos valores están por encima de los encontrados por González et al. (2002) quienes reportaron un Kc máximo de 0,82 . Diámetros muy delgados afectan el soporte de la planta, lo que puede incidir en la capacidad fotosintética de la planta. Sin embargo, se muestra una tendencia a incrementar el diámetro de tallo al aumentar el coeficiente multiplicador, similar a lo encontrado por Marín (2010) en plantas de cilantro. De acuerdo con Fronza y Vinícius (2003) valores de Kc entre 1,16 y 1,45, fueron los que mejores respuestas agronómicas presentaron en las plantas de estevia. No obstante, Daza et al. (2015) reportaron que el uso de coeficientes de 1,2 puede inducir pérdidas de nitratos por lixiviación y no alcanzar los máximos rendimientos.

La mayor área foliar fue obtenida con el coeficiente de 1,2 pero sin encontrar diferencias significativas con los demás tratamientos debido a la alta dispersión de los datos. Sin embargo, este coeficiente estuvo 18, 30,5;
13,8 y $4,3 \%$ por encima de los coeficientes de 0,$9 ; 1,0 ; 1,1$ y 1,3 respectivamente. A mayor área foliar, mayor posibilidad de realización del proceso fotosintético lo que permite un mayor crecimiento y desarrollo de la planta (Delgado 2012).

Los porcentajes de contenido relativo de agua estuvieron entre 23 y $34,0 \%$ sin diferencias significativas entre los diferentes coeficientes (Figura 1). El coeficiente multiplicador de 1 estuvo entre 24 y $34 \%$ por encima de los demás coeficientes mostrando con el suministro de la lámina de agua calculada con este coeficiente mostró la mejor eficiencia de almacenamiento y retención en las hojas de Estevia. Similares resultados fueron hallados por Delgado (2012) quien encontró una correlación negativa entre área foliar y contenido relativo de agua en las hojas de albahaca y lo atribuye al hecho de que a mayor área foliar, mayor área efectiva de pérdida de agua por estomas y por lo tanto, menor capacidad de retención de agua en los tejidos. 


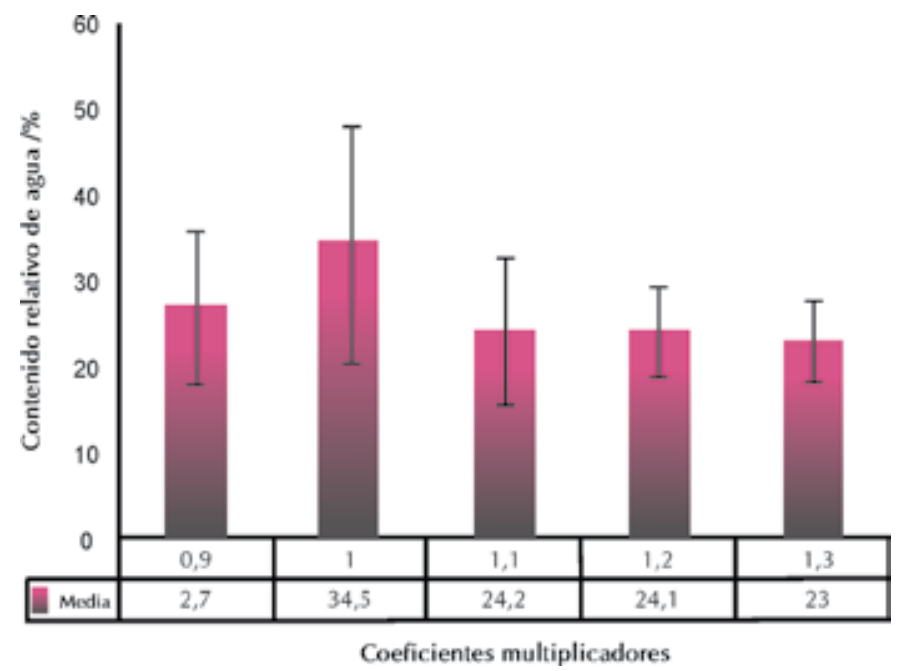

Figura 1. Efecto de la lámina de riego calculada a partir de la variación de coeficientes multiplicadores de la evapotranspiración en el contenido relativo de agua en las hojas de Estevia (Stevia Rebaudiana Bert.). Las barras indican el intervalo de confianza para la media al 95\%. Prueba de Tukey $F=1,39(p=0,253)$

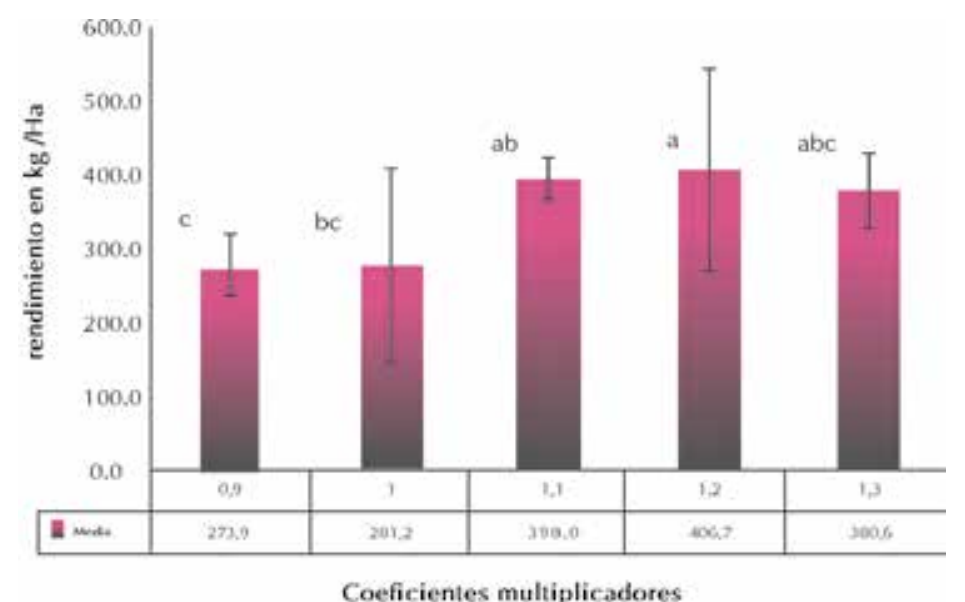

Figura 2. Efecto de la lámina de riego calculada a partir de la variación de coeficientes multiplicadores de la evapotranspiración en el rendimiento de Estevia (Stevia Rebaudiana Bert.). Las barras indican el intervalo de confianza para la media al 95\%. Prueba de Tukey $F=5,27$ ( $p=0.007)$.

El coeficiente de 1,2 fue el que obtuvo el mayor rendimiento de hojas de Estevia por unidad de área, el cual estuvo por encima de $400 \mathrm{~kg} \mathrm{ha}^{-1}$ (Figura 2). Este rendimiento estuvo 32,6; 30,9; 2,14 y $6,3 \%$ por encima de los rendimientos alcanzados por los coeficientes 0,$9 ; 1,0$; 1,1 y 1,3 respectivamente. Estos resultados estuvieron por debajo de los encontrados por Yang et al. (2013) quienes alcanzaron rendimientos máximos de 4,5 $\mathrm{Mg} \mathrm{ha}^{-1}$ pero fueron similares a los de Espitia et al. (2009) quienes en la región media del Sinú reportaron rendimientos entre 3,6 y 3,8 $\mathrm{Mg} \mathrm{ha}^{-1}$. No se encontraron diferencias significativas entre coeficientes más altos en el rendimiento alcanzado, por lo que es posible que los bajos rendimientos estén asociados a factores diferentes al riego como la fertilización 
o las condiciones climatológicas donde se llevó el experimento. Niño et al. (2013) encontraron que láminas de riego superiores a 5,4 mm no incrementaron el índice de cosecha ni la concentración de edulcorantes en el cultivo de Estevia.

\section{CONCLUSIONES}

No se encontraron diferencias significativas entre las láminas de riego evaluadas en el cultivo de Estevia. Sin embargo, las tendencias de los resultados muestran que el coeficiente de 1,2 es el que mejores respuestas presentó en la mayoría de las variables agronómicas evaluadas. La aplicación de la lámina de riego calculada con este coeficiente obtuvo mejores resultados en especial en la etapa de crecimiento en comparación con los demás coeficientes, pero los resultados agronómicos obtenidos están por debajo de lo reportado por otros autores, lo que demuestra la posibilidad de que este valor este por debajo del coeficiente del cultivo (Kc). A pesar de lo anterior la determinación de este coeficiente es un valor de partida para el cálculo de láminas de riego en sitios donde no se cuenta con información suficiente para realizar los balances hídricos necesarios para la determinación del Kc.

\section{REFERENCIAS}

Allen, R., Pereira, L., Raes, D. y Smith, M. 2006. Evapotranspiración del cultivo. Guía para la determinación de los requerimientos de agua de los cultivos. Organización de las Naciones Unidas para la agricultura y la alimentación FAO. Estudio Riego y Drenaje 56, Roma, 300 p.
Álvarez, J, Balanguera, L. y Pardo, C. 2010. Efecto de la propagación de la aplicación de diversas laminas y frecuencias de riego en la propagación del romero (Rosmarinus officinalis L.). Rev. Ing. Invest. 30 (1):86-90.

Argentel, L., González, L.M., Ávila, C. y Aguilera, R. 2006. Comportamiento del contenido relativo de agua $y$ la concentración de pigmentos fotosintéticos de variedades de trigo cultivadas en condiciones de salinidad. Cultivos Tropicales 27(3):49 -53.

Casaccia, J. y Álvarez, E. 2006. Recomendaciones técnicas para una producción sustentable del ka'a he'e (Stevia rebaudiana Bertoni) en el Paraguay. Ministerio de Agricultura y Ganadería Subsecretaría de Estado de Agricultura, Dirección de Investigación Agrícola, Caacupe, Paraguay, p9-50.

Casierra, F. y Vargas, Y. 2007. Crecimiento y producción de fruta en cultivares de fresa (Fragaria sp) afectadas por encharcamiento. Rev. Col. Ciencias Hort. 1(1):21 -32.

Cuervo, J.L. 2012. Manual de los cultivos de albahaca, menta y Estevia. Facultad de Agronomía, Universidad Nacional de Colombia, Bogotá.

Daza, M., Díaz, J., Aguirre, E. y Urrutia, N. 2015. Efecto de abonos de liberación lenta en la lixiviación de nitratos y nutrición nitrogenada en estevia. Revista colombiana de ciencias hortícolas. 9(1):112-123. 
Delgado, G. 2012. Determinación de la lámina de riego para el cultivo de la Albahaca Genovesa (Ocimum basilicum "Genovese".) a partir de la variación del coeficiente multiplicador de la evapotranspiración. Tesis Ingeniero Agrícola, Universidad del Valle, Santiago de Cali.

EEA - European Environment Agency. 2012. Towards efficient use of wáter resources in Europe. Rosendahls-Schultz Grafisk. Copenhagen p12.

Espitia, M., Montoya, R. y Atencio, L. 2009. Rendimiento de Stevia rebaudiana Bert. Bajo tres arreglos poblacionales en el sinú medio. Rev. UDCA Actualidad y Divulgación científica 12(1):151-161.

Fronza, D y Vinícius, M. 2003. Water consumption of the Estevia (stevia rebaudiana (Bert.) Bertoni) crop estimated through microlysimeter. Scientia Agrícola 60(3): 595-599.

González, R., Paniagua, J. y Mayeregger, E. 2002. Necesidad de agua para el cultivo de Estevia (stevia Rebaudiana Bert.), calculado sobre la base de lectura de microlisímetro. Investigación Agraria 4(2):19-24.

Hadid, A. 2004. Proposal for Expanding the Crop Mandate of ICARDA to Include Horticultural Crops. ICARDA, Syria, p17-19.
Jarma, A., Rengifo, T. y Aramendiz, H. 2005. Aspectos fisiológicos de Estevia (Stevia rebaudiana Bertoni) en el Caribe colombiano: I Efecto de la radiación incidente sobre el área foliar y la distribución de biomasa. Agronomía Colombiana 23(2):207-216.

Lavini, A., Riccardi, M., Pulvento, C., De Luca, S., Scamosci M. y d'Andria. R. 2008. Yield, quality and water consumption of Stevia rebaudiana Bertoni grown under different irrigation regimenes in Southern Italy. Ital. J. Agron. 2:135-143.

Marín, G. 2010. Determinación de los requerimientos hídricos del cilantro (Coriandrum sativum), variedad unapal precoz y su relación con el desarrollo del cultivo, la producción y la calidad, comparando un periodo seco y húmedo de siembra del cultivo en el año. Tesis Ingeniero Agrónomo, Universidad Nacional de Colombia, Palmira.

Martìnez, L. 2010. Estudio de la calidad agronómica del agua de riego de las islas Baleares. Conselleria de Medio Ambiente y Movilidad - Dirección General de Recursos Hídricos, Instituto Geológico y Minero de España (IGME), Madrid. p7-9.

Niño, J., Cuervo, J. y Villalobos, R. 2013. Efecto del potencial mátrico del suelo en el crecimiento del cultivo de Estevia (Stevia rebaudiana Bert). Rev. Col. Cienc. Hort. 7(2): 240-251. 
ONU - Organización de las Naciones Unidas. 2011. Agua y agricultura en la economía verde. Programa ONU agua para la promoción y la comunicación en el marco del decenio. Disponible en: http:// www.un.org/spanish/waterforlifedecade/ green_economy_2011/pdf/info_brief_ water_and_agriculture_spa.pdf.

Silva, R. 2002. Efeito do estresse hídrico sobre características de crescimento e a produção de óleo essencial de Melaleuca alternifolia Cheel. Acta Scient. 24: 13631368.

\section{Vera, J., Grageda, O., Vuelvas, M. y Peña, J.} 2001. Absorción de nitrógeno (15n) por el cultivo de cebada en relación con la disponibilidad de agua En "El Bajío", Guanajuato, México. Terra 20:57-64.
Wilches, F. y Álvarez, J. 2007. Tamaños de alveolo y diferentes láminas de riego en obtención de plántulas de tomate (Lycopersicon esculentum Mill). Tesis Ingeniero Agrónomo, Universidad Pedagógica y Tecnológica de Tunja, Tunja.

Yang, J., Liu, X. y Shi, Y. 2013. Effect of Different Mixed Fertilizer on Yield, Quality and Economic Benefits in Stevia rebaudiana Bertoni. J. Food Sci. Techn. 5(5):588591. 